it meant that free bus travel was typically held by all of a peer group. This rendered buses a socially inclusive way for groups to travel and spend time together, thereby enhancing group-level capabilities.

Conclusion We believe this attention to individual and group-level capabilities for self-determination provides the basis for a broader and more child-centered view of independent mobility than is typi$\mathrm{cal}$ in health research. The importance of the universal nature of the entitlement to free bus travel also provides an example of how policy interventions with universal coverage may have effects which are more than the 'sum of the parts' of alternative, targeted approaches.

\section{PSO3 SYSTEMATIC REVIEW AND META-ANALYSIS OF SCHOOL- BASED INTERVENTIONS TO REDUCE BODY MASS INDEX}

doi:10.1136/jech-2012-201753.102

HV Lavelle, JP Pell, DF Mackay. Centre for Population Health Sciences, University of Glasgow, Glasgow, UK

Background Childhood obesity predisposes to adult obesity and increases the risk of many diseases. Schools provide a vehicle to deliver public health interventions to all children.

Methods Medline and Embase were used to undertake a systematic review of published studies on school-based interventions aimed at reducing body mass index (BMI) of children $\leq 18$ years. Preferred reporting items for systematic review and meta-analyses guidelines were followed, and eligible studies subjected to a random effects meta-analysis.

Results Between 1991 and 2010, 43 published studies provided 60 measurements of effect. The pooled effect was a 0.17 (95\% CI: 0.08 , $0.26, P<0.001)$ reduction in BMI. Heterogeneity was high $\left(I^{2}=93.4 \%\right)$ but there was no significant small study bias (Egger's test, $P=0.422$ ) nor significant variation by length of follow-iup. The intervention comprised physical activity only in $11(26 \%)$ studies, education only in three (7\%), and combinations of these and improved nutrition in the remaining 29 (67\%). On stratified analysis, physical activity used in isolation $(-0.13,95 \% \mathrm{CI}:-0.22,-0.04, P=0.001)$ or combined with improved nutrition $(-0.17,95 \%$ CI: $-0.29,-0.06, P<0.001)$ was associated with significant improvements in BMI. Interventions targeted at overweight/obese children reduced their BMI by 0.35 ( $95 \%$ CI: $0.12,0.58, P=0.003$ ). Those delivered to all children reduced it by 0.16 (95\% CI: $0.06,0.25, P=0.002)$.

Conclusion There is growing evidence that school-based interventions that contain a physical activity component may be effective in helping to reduce BMI in children.

\section{PSO4 VALIDITY OF CANCER DIAGNOSIS IN A PRIMARY CARE DATABASE COMPARED WITH LINKED CANCER REGISTRATIONS IN ENGLAND. POPULATION-BASED COHORT STUDY}

doi:10.1136/jech-2012-201753.103

${ }^{1} \mathrm{~A}$ Dregan, ${ }^{2 T}$ Murray-Thomas, ${ }^{3} \mathrm{H}$ Moller, ${ }^{1} \mathrm{M}$ Gulliford. ${ }^{1}$ Primary Care and Public Health Sciences, King's College London, London, UK; ${ }^{2}$ General Practice Research Database Group, MHRA, London, UK; ${ }^{3}$ Thames Cancer Registry, King's College London, London, $U K$

Background Electronic health records from primary care are increasingly utilised as a resource for epidemiological research in cancer. The present study aimed to evaluate the validity of cancer diagnoses recorded in a primary care database compared with cancer registry data in England.

Methods Eligible cancer codes were reviewed and agreed by two epidemiologists with experience in cancer and primary care. The eligible cohort comprised 42,556 participants, registered with English general practices in the General Practice Research Database
(GPRD) that consented to cancer registry linkage, who presented with haematuria, haemoptysis, dysphagia or rectal bleeding or were diagnosed with cancer of the lung, urinary tract, oesophagus/stomach, or colon/rectum between 2002 and 2006. Cancer registry (CR) records were linked through a unique identifier by a third party. Cancer registry and primary care records were compared for cancer diagnosis, date of cancer diagnosis and death.

Results There were 5,429 cancer diagnoses in GPRD and 5,710 in the CR, with 5,216 (91\% of CR total) diagnosed in both sources. There were 494 (9\%) diagnosed in CR but not in GPRD and 213 (4\%) that were diagnosed in GPRD but not CR. The predictive value of a GPRD cancer diagnosis was $96 \%$ for lung cancer, $92 \%$ for urinary tract cancer, $96 \%$ for gastro-oesophageal cancer and $98 \%$ for colorectal cancer. 'False negative' primary care records were sometimes accounted for by registration end dates being shortly before cancer diagnosis dates. The median (interquartile range) difference in date of cancer diagnosis (CR minus GPRD) was -11 (-30 to 6) days. Death records were consistent for the two sources for 3,337/3,397 (99\%) of cases.

Conclusion Recording of cancer diagnosis and mortality in primary care electronic records is generally consistent with cancer registrations in England. Linkage studies must pay careful attention to selection of codes to define eligibility and timing of diagnoses in relation to beginning and end of record.

\section{PSO5 MEN WITH PROSTATE CANCER MAKE POSITIVE DIETARY CHANGES FOLLOWING TREATMENT IN A RANDOMISED TRIAL: A PROSPECTIVE COHORT STUDY}

doi:10.1136/jech-2012-201753.104

${ }^{1} \mathrm{KNL}$ Avery, ' $\mathrm{JL}$ Donovan, 'R Gilbert, 'M Davis, 'P Emmett, 'E Down, ${ }^{2} \mathrm{~S}$ Oliver, ${ }^{3} \mathrm{DE}$ Neal, ${ }^{4} \mathrm{FC}$ Hamdy, ${ }^{\mathrm{J} A}$ Lane. ' $S$ School of Social and Community Medicine, University of Bristol, Bristol, UK; ${ }^{2}$ Department of Health Sciences, University of York, York, UK; ${ }^{3} \mathrm{Oncol}$ ogy Centre, Addenbrooke's Hospital, Cambridge, UK; ${ }^{4}$ Department of Surgical Sciences, University of Oxford, Oxford, UK

Background Prostate cancer (PC) is the second most frequently diagnosed cancer of men worldwide. A healthy diet may improve clinical outcomes but there is currently no disease-specific dietary advice available to aid survivorship after a PC diagnosis. The effect of a PC diagnosis on men's diet remains uncertain because, although cancer survivors report dietary changes, the direction and magnitude of changes are poorly understood. This study examined dietary changes in men before and after treatment for PC within the Prostate Testing for Cancer and Treatment (ProtecT) randomised trial.

Methods This was a prospective cohort study embedded within the Protec T randomised trial of treatments for PC. Participants were men aged 50-69 years tested for PC in primary care centres in nine areas of the UK. 3935 men completed a validated food frequency questionnaire before diagnosis and 678 with localised disease repeated the questionnaire one year later (response $82.7 \%$ ) Pre-diagnosis dietary intakes of men with different diagnoses subsequently (negative diagnosis, at risk, localised PC, advanced PC) were compared using linear regression. Dietary changes after a diagnosis of localised PC were examined using non-parametric tests. Effects of cancer treatments (radical prostatectomy, conformal external beam radiotherapy or active monitoring) were examined using linear regression.

Results Before diagnosis, all men consumed largely similar amounts of key nutrients and foods. The diagnosis of localised PC led to dietary changes in $29.2 \%$ of men. Changes observed were generally in a healthy direction, with 234 (34.7\%) men eating more fresh tomatoes $(\mathrm{p}<0.0001)$ and $156(23.5 \%)$ more tomato products $(p=0.01) .271(40.0 \%)$ men consumed more protein $(p<0.0001)$ and $193(28.6 \%)$ more fruit/vegetable juice $(p<0.0001)$. Less macronutrients were obtained from dairy products $(p<0.01)$. Men undergoing 
active monitoring (regular assessment of disease status) drank more fruit/vegetable juice than men who had received surgery $(p=0.004)$. Conclusion Diagnosis of localised PC prompted around one third of men to adopt healthier diets and eat more 'prostate-healthy' foods. Dietary choices were influenced by treatment received, with more interest in dietary factors for survivorship in men undergoing active monitoring than those undergoing radical treatment. PC survivors are motivated to improve their diet and are able to access and act on healthy eating advice. Diagnosis of PC may serve as a "teachable moment", thereby providing opportunities for clinicians to provide targeted advice that could be beneficial to general (and prostate-specific) health and to support survivorship.

\section{PS06 FINDING THE MISSING UNITS: IDENTIFYING UNDER- REPORTING OF ALCOHOL CONSUMPTION IN ENGLAND}

doi:10.1136/jech-2012-201753.105

S Boniface. Epidemiology and Public Health, UCL, London, UK

Background Alcohol sales coverage (reported consumption as a percentage of total sales) is typically $40-60 \%$. This study explores how accidental under-estimation of alcohol in drinks poured at home might contribute to low sales coverage.

Methods 283 drinking adults (16+) completed a face-to-face survey comprising a questionnaire and pouring task on 12 occasions at six sites in and near London in summer 2011. Actual and perceived number of units poured in a 'usual glass' of wines and spirits is explored by demographic and social factors.

Results There were 283 participants (54\% women) who completed the questionnaire and pouring task. The 283 participants poured a total of 465 glasses of wine and spirits. $52 \%$ wine estimates and $42 \%$ spirit estimates were within 0.5 units of their actual value. More participants over-estimated the amount of alcohol they had poured than under-estimated. Multinomial logistic regression found significant risk factors for under-estimating wine were increasing volume of wine poured (RRR $1.0295 \%$ CI 1.01-1.02, $\mathrm{P}<0.0001$ ), belonging to a non-white ethnicity $(\mathrm{RRR} 3.88,1.65-9.16, \mathrm{P}=0.002)$, and being unemployed or retired ( $R R R$ 4.30, 1.08-17.07, $\mathrm{P}=0.038$ ). Only the volume of spirits poured was a significant predictor of under-estimating spirits ( $R R R$ 1.04, 1.01-1.06, $\mathrm{P}=0.003$ ). For both wines and spirits, not having a degree was significantly associated with an increased risk of over-estimating the amount of alcohol poured.

Conclusion As participants were more likely to over-estimate than under-estimate, this survey is not able to explain low sales coverage as a product of accidental under-estimation of the amount of alcohol poured drinks drunk at home. A future qualitative study will explore how recall accuracy may contribute to low sales coverage.

\section{PS07 TRENDS IN BLO0D PRESSURE IN ENGLAND: G00D TREATMENT OR GOOD LUCK?}

doi:10.1136/jech-2012-201753.106

SJ DeWilde, IM Carey, SM Shah, T Harris, AR Rudnicka, PH Whincup, DG Cook. PHSE, St George's, University of London, London, UK

Background For 30 years, adult blood pressure (BP) has declined in many developed countries. This is likely to have contributed to declining cardiovascular disease mortality. However, the reasons for this, particularly the impact of increased antihypertensive treatment, have been little studied. Diagnostic criteria have changed, meaning that more patients are treated at lower levels of BP than previously; and treatment intensity is greater. Accounting for the effects of treatment on BP trends is complex and open to misinterpretation.

Methods Using the Health Survey for England we examined BP measures and treatment in white subjects aged 18+ years over two periods, 1994-2002 and 2003-2009. To examine trends independent of anti-hypertensive treatment, untreated BP was estimated from the recorded BP on treatment. To do this, a model was derived using published data on the effect of anti-hypertensives used singly and in combination at differing pre-treatment BP levels. BP untreated with statins was similarly estimated.

Results Among an average 9,147 subjects per year, mean systolic BP (SBP) in men declined by $0.55 \mathrm{mmHg}$ annually $(95 \%$ CI 0.46 to 0.64 ) between $1995-2002 ; 0.34 \mathrm{mmHg}$ annually (95\% CI 0.21 to 0.46 ) between 2003-2009. Similar trends occurred in women. The decline was greater in older groups, but present in young, largely untreated groups, although the trend was greatly reduced in young men by 2009. Trends in estimated SBP adjusting for the influence of treatment, where present, exhibited only slightly lower declines; for men: $0.47 \mathrm{mmHg}$ annually (95\% CI 0.37 to 0.57 ) between 1995 2002; $0.27 \mathrm{mmHg}$ annually (95\% CI 0.13 to 0.41 ) between 2003 2009. Statin therapy had little additional effect. Diastolic blood pressure showed similar but weaker trends.

Conclusion For 15 years, BP declined in English adults. The overall decline in SBP of nearly $5 \mathrm{mmHg}$ over the study period is likely to be of clinical significance in reducing CVD events. For an individual aged $40-49$ a $5 \mathrm{mmHg}$ reduction in SBP, as seen here, would be expected to reduce the risk of stroke by $23 \%$ and of IHD by $16 \%$. It is therefore of concern that, in recent years, the decline has essentially ceased in the youngest age groups, particularly in younger men. The effect of treatment was modest; less than $25 \%$ of the male SBP decline is attributable to it. Other explanations for this fall, occurring whilst obesity has increased, need further exploration, but reduced salt intake is a likely candidate.

\section{PSO8 IS THERE A PROTECTIVE EFFECT OF HIGH MINERAL CONTENT IN DRINKING WATER ON CORONARY HEART DISEASE MORTALITY? A CROSS-SECTIONAL ECOLOGICAL STUDY OF A LARGE POPULATION IN CENTRAL ENGLAND}

doi:10.1136/jech-2012-201753.107

GM Rudge, MA Mohammed. School of Health and Population Sciences, University of Birmingham, Birmingham, UK

Background It has been hypothesised that dissolved minerals in water, particularly calcium carbonate and magnesium carbonate, are protective against heart disease. Animal studies suggest biologically plausible mechanisms for this and statistically significant effects have been presented from several large ecological studies.

Setting The West Midlands Government Office Region in central England, has a large and diverse population which has great variation in water hardness over a relatively small area. Our units of analysis were West Midlands neighbourhoods (lower level super output areas) falling in areas supplied by two water companies. We obtained exposure estimates from water industry measurements taken in 2007.

Methods Using geographical information system software, digitised supply water supply maps were mapped to our neighbourhood geography. Having established the distribution of water hardness through neighbourhoods, we mapped tertiles of hardness and identified neighbourhoods inside them. To these we linked emergency myocardial infarction (MI) admissions for 45-74 year-old residents for a three year period, adjusting for the age distribution of the neighbourhoods, ethnic mix and socio-economic deprivation. We used a negative binomial model to determine the degree of association between water hardness and MI admission counts, adjusting for the other variables.

Results We were able to accurately determine the mineral content of water supplied to 2,925 neighbourhoods with a total population of over 4.5 million. Contrary to other studies, we found just a small but non-significant negative correlation between hardness and MI admissions were seen in men, with an incidence rate ratio (IRR) of 0.97 , per tertile, $(0.92-1.03, \mathrm{p}=0.37)$ and a small, non-significant positive one seen in women, IRR=1.02, $(0.93-1.12, p=2)$. 